\title{
ESTRATEGIAS METODOLÓGICAS PARA LA ENSEÑANZA DE LA MATEMÁTICA UTILIZANDO TECNOLOGÍA
}

\section{METHODOLOGICAL STRATEGIES FOR THE TEACHING OF MATHEMATICS USING TECHNOLOGY}

\section{RESUMEN}

\author{
Karol Menay ${ }^{1}$; Jaime Leiva N. ${ }^{2}$
}

El trabajo realizado corresponde a una experiencia de enseñanza aprendizaje en el área de matemática, mediada por TIC específicamente el blog y videos que consideró como base el método de aprendizaje entre pares. Si bien el método señalado esta descrito para parejas de estudiantes, en este caso se realizó entre cursos paralelos, es decir el curso A construyó materiales para el curso B y viceversa. Para tales efectos se tuvo que capacitar al profesor en el manejo de blog y a los estudiantes en la edición de videos. Los temas seleccionados para la experiencia fueron trigonometría y cónicas. Los resultados muestran que los estudiantes trabajaron motivados y con la expectativa de que los videos que su curso generaron realmente sirvieran para que los estudiantes del otro curso aprendieran mejor los temas señalados. Todos se mostraron abiertos a usar las tecnologías y consideraron que era una estrategia más llamativa y entretenida para aprender. Por otra parte el profesor consideró interesante explorar estas tecnologías para modificar los métodos de enseñanza aunque considera que debe estar mejor capacitado y que requiere mayor tiempo y perfeccionamiento para incorporarlas con más soltura y naturalidad. En términos globales debemos señalar que la experiencia fue bien valorada en el establecimiento, al punto que han decidido implementar us sistema similar, apoyado en tecnología de internet para la asignatura de matemática en todos los niveles.

Palavras-Chave: Metodología, Aprendizaje entre pares, estrategias, aula, innovar

\begin{abstract}
The work carried out corresponds to a teaching-learning experience in the field of mathematics, mediated by technology specifically the blog and videos that considered as a basis the method of peer learning. Although the aforementioned method is described for pairs of students, in this case it was carried out between parallel courses, that is, course A constructed materials for course B and vice versa. For such effects, the teacher had to be trained in the management of the blog and the students in the edition of videos. The topics selected for the experience were trigonometry and conics. The results show that the students worked motivated and with the expectation that the videos that their course generated really served so that the students of the other course learned better the indicated subjects. All were open to using the technologies and considered it a more eye-catching and entertaining strategy to learn. On the other hand the professor considered interesting to explore these technologies to modify the methods of education although it considers that it must be better trained and that it requires more time and improvement to incorporate them with more ease and naturalness. In global terms we must point out that the experience was well valued in the establishment, to the point that they have decided to implement their similar system, supported by internet technology for the mathematics subject at all levels.
\end{abstract}

Keywords: Methodology, Peer learning, strategies, classroom, innovate

\footnotetext{
${ }^{1}$ Matemática, Desarrollo páginas web, Iuniversidad de Playa Ancha, e-mail

2 Profesor, Universidad de Playa Ancha, jleiva@upla.cl
} 


\section{INTRODUCCIÓN}

Involucrarse con los nuevos medios digitales nos da la posibilidad de participar y generar actividades interactivas y colaborativas. El día a día está lleno de experiencias relacionadas con aparatos electrónicos pensados para facilitarnos la vida, y el sistema educativo no puede dejar de lado la gran importancia que tienen si pretende conseguir un desarrollo integral de los alumnos. Es importante hacer visible el uso de las tecnologías al interior de los establecimientos educacionales, con el fin de probar de qué forma pueden ser utilizadas para mejorar los aprendizajes de los estudiantes, más aún, considerando que la penetración de internet y el uso de las redes en el mundo crece de manera exponencial. Un informe reciente de la National School Boards Association de los Estados Unidos, que atiende a cuarenta y siete millones de alumnos, descubrió que el $96 \%$ de los estudiantes con acceso a Internet usan herramientas de interacción social con texto, imágenes y video. (Llorente \& Iglesias, 2018). Según la información proporcionada por Internet World Stats (2017) en base a datos proporcionados por organizaciones líderes en investigaciones de Internet (Nielsen / NR, eTForcasts, CIAlmanac, ITU, IWS, CIA), a partir de marzo de 2017, el número de usuarios de Internet en todo el mundo aumentó aproximadamente 3 mil millones 732 millones de personas (49.6\%). La tasa de aumento en el uso de internet 2000-2017 fue de 933.8\%.

\section{FUNDAMENTACIÓN TEÓRICA}

\section{La tecnología blog}

Los Blog son una de las tecnologías más populares en el mundo de internet y es una de las primeras herramientas de publicación que permiten crear y publicar una bitácora en línea. Existe gran cantidad de servicios gratuitos que ofrecen herramientas y alojamiento para mantener un blog sin necesidad de poseer un servidor propio. Estos servicios proporcionan todo lo necesario para empezar a escribir y publicar en un instante (Orihuela, 2006), no teniendo el usuario que escribir ningún código o instalar programas de servidor.

Cualquier usuario de Gmail, tiene acceso directo a Blogger, aplicación gratuita que brinda un espacio a los usuarios de internet para publicar su propio contenido y compartirlo con sus seguidores, lo que se puede realizar utilizando cualquier navegador web actualizado y los resultados se reflejan inmediatamente (Peña, Córcoles, \& Casado, 2006). Se pueden crear varios blog, pues el servicio no pone límites en ese sentido. Blogger contiene plantillas prediseñadas de excelente calidad, con infinidad de modelos, gadgets y colores. Esta herramienta puede ser muy útil e innovadora para los docentes, ya que a través de un blog pueden organizar sus clases y cursos, mantener actualizados los contenidos para sus estudiantes, entregar noticias y desarrollar actividades propias de su asignatura. En definitiva, un blog es una bitácora en línea que se puede convertir en un instrumento ideal para que los estudiantes se motiven y esfuercen por mejorar su formación, sacando lo mejor de ellos mismos y, naturalmente, lo mejor de su expresión escrita (Meneses \& Regaña, 2008). Todo a través de un ambiente más interactivo donde pueden participar el profesor y los estudiantes

\section{Blog como herramienta educativa}


Los blogs han ganado una creciente popularidad en los últimos años, extendiéndose a todas las esferas del ámbito social, incluidas las iniciativas en el campo de la formación. (Meneses, 2009). Una de las características es la configuración cronológica inversa de las entradas, y en el que se recogen, a modo de diario, enlaces, noticias y opiniones de autoría mayoritariamente individual con un estilo informal y subjetivo (Barujel, 2005).

Hoy en día es fundamental que los docentes tengan un espacio en la web para trabajar con sus estudiantes usando los medios digitales disponibles. El uso de blog permite al profesor publicar contenidos, recursos, enlaces y dejar sus experiencias para que otros docentes puedan usarlos en sus aulas.

En los últimos años, son numerosas las experiencias que se han generado en todos los niveles educativos con herramientas tecnológicas, y muchos docentes han elaborado Edublogs como guía de los procesos de enseñanza aprendizaje, encaminadas en su mayoría a la construcción de webs personales dinámicas, para compartir ideas y proyectos, o como un instrumento de soporte para los contenidos curriculares (Llorente \& Iglesias, 2018). Los blogs, y en general las tics han mostrado tener un alto potencial dentro de la educación infantil, como parte del desarrollo, identificando patrones de texto, video e imágenes, y componiendo, en función de ello, diapositivas individuales que contienen un único tipo de información, ya sea blogs de animaciones infantiles, educación infantil, muestras, recursos para educación infantil, etc. $((«$ Esos locos bajitos de infantil», s. f.),(«Mil recursos educación infantil», s. f.) ,(«Recursos para educación infantil», s. f.) ,(«Actividades para Educación Infantil», s. f.)). En la educación superior se manifiesta la necesidad de ayudar y potenciar la constitución y consolidación de equipos de trabajo, facilitar el diálogo e intercambio de ideas, experiencias entre los diferentes miembros de la comunidad universitaria, la indagación e investigación educativa, la reflexión colectiva, la interacción y la participación social con su entorno familiar y profesional.

\section{Estrategias de aprendizaje con Tic}

Hoy, las TIC están generando un impacto importante en las estrategias de enseñanza que utilizan los docentes con sus estudiantes ya que se hace evidente la necesidad de incorporar estas herramientas como parte de su quehacer, reconociendo las potencialidades de estos recursos y generando nuevos enfoques educativos para su uso. Parte de este impacto es el gran número de estrategias pedagógicas que han surgido en los últimos años, muchas de las cuales se asocian al uso de tecnologías específicas y se potencian entre ellas cuando se utilizan de manera apropiada: aprendizaje activo, aprendizaje auténtico, aprendizaje basado en la investigación, aprendizaje basado en problemas, aprendizaje basado en proyecto, aprendizaje basado en retos, aprendizaje colaborativo, aprendizaje en línea, aprendizaje flexible, aprendizaje híbrido, aprendizaje invertido, aprendizaje justo a tiempo, aprendizaje vivencial, aprendizaje-servicio, entornos de aprendizaje autorregulados, gamificación, aprendizaje entre pares (Radar de Innovación Educativa, 2017)

El nuevo sistema demanda herramientas que faciliten el trabajo cooperativo y mejoren la gestión del conocimiento, a modo de módulos anexos o integrados dentro de plataformas de elearning. (Bauerová \& Sein-Echaluce, 2007), con el fin de que el docente pueda facilitar el apoyo a las actividades educativas, fuentes y herramientas, y así fomentar el trabajo de los estudiantes, motivarlos y atender sus individualidades. En la actualidad, la buena calidad de los contenidos de un curso, en el sentido de fuente rica de información, es el criterio más importante que determina la distinción del mismo. Sin embargo, en el nuevo modelo la calidad se mide predominantemente por el tipo de trabajo de los estudiantes y por cómo el profesor los conduce. Además, la comprobación del rendimiento del trabajo del alumno viene siendo la guía principal de su aprendizaje, mientras que la base de su progreso debería enfocarse a estimular la automotivación. 
Los estilos de aprendizaje y el aprendizaje entre pares están totalmente relacionados, ya que facilitan la atención de diversidad y la mejora de procesos del alumnado; como señalan Loke y Chow (2007), los estudiantes que participan en los procesos de aprendizaje entre iguales mejoran sus habilidades de aprendizaje y su capacidad reflexiva de pensamiento crítico, lo que implica una mejora en su EA. De la misma forma, un fortalecimiento de los Estilos de Aprendizaje de los estudiantes puede mejorar las experiencias con otros de sus pares (Rodríguez y Vázquez, 2013) y, con ello, avanzar en la educación entre iguales. Por lo general, el aprendizaje cooperativo se comprende como un proceso caracterizado por la participación activa, responsable, intencional y articulada de cada uno de los miembros de un grupo, quienes pretenden contribuir al desarrollo de habilidades de los demás y de sí mismos (Domingo, 2008; Johnson, Johnson \& Holubec, 1999); además de perseguir una meta común o el desarrollo de un producto final (Panitz,1999).(Chois-Lenis, Casas-Bustillo, LópezHiguera, Prado-Mosquera, \& Cajas-Paz, 2017).

\section{METODOLOGÍA}

La investigación se enmarca en un paradigma positivista, con alcance cuantitativo, de medición transversal, en un contexto propositivo. Se desarrolló con un total de 67 estudiantes, de los cursos 3ro medio B y 3ro medio C del colegio Casteliano en la asignatura de matemática. Se basa fundamentalmente en el uso de las TIC en la actividad docente del profesor de matemática dentro del aula, integrándolas a su planificación anual como nuevas alternativas metodológicas para el aprendizaje de los estudiantes. Específicamente se utilizará un blog para colocar contenidos creados por los estudiantes. Para el desarrollo del trabajo se consideraron las siguientes etapas:

\section{Etapa 1: Capacitación}

Se capacitó al profesor para crear mantener y usar un blog considerando los siguientes aspectos: crear un blog, modificar la estética del blog, creación de entradas con contenidos en diferentes formatos (texto, imágenes, videos, pdf), usar gadget, crear páginas estáticas y manejar el panel de administración.

Etapa 2: Selección de contenido

Se trabajó con el profesor en la selección de los contenidos del curso que el estimaba podrían ser apoyados con uso de tecnología, específicamente el blog.

Etapa 3: Diseño de estrategia metodológica

El profesor seleccionó los temas de trigonometría y cónicas, donde los estudiantes deberán realizar algunos videos y un trabajo escrito con la temática dada por el profesor respectivamente y se creará el primer blog de matemática del colegio con material creado por el profesor y los propios estudiantes. Se definió que el Tercero B generará materiales para el tema de Cónicas y el Tercero C para Trigonometría (Esquema 1). Los materiales creados serán incorporados por el profesor en el proceso 
de aprendizaje de del curso contrario .

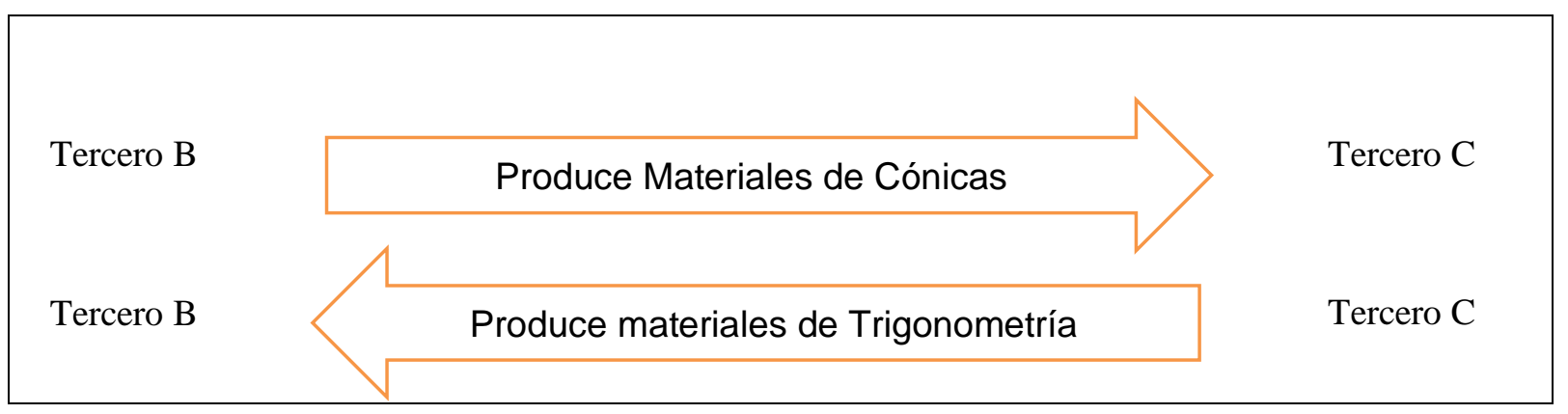

Esquema 1: Forma de trabajo de los cursos

Etapa 4: Desarrollo de la experiencia

Para el desarrollo de la experiencia el profesor del curso generó un blog que llamó

"MatematicaCasteliano" en la dirección https://matematicasteliano.blogspot.com/. Las primeras entradas del blog fueron las necesarias para explicar a los estudiantes la forma de trabajar y entregar información general que los oriente durante el proceso, ver imagen 1

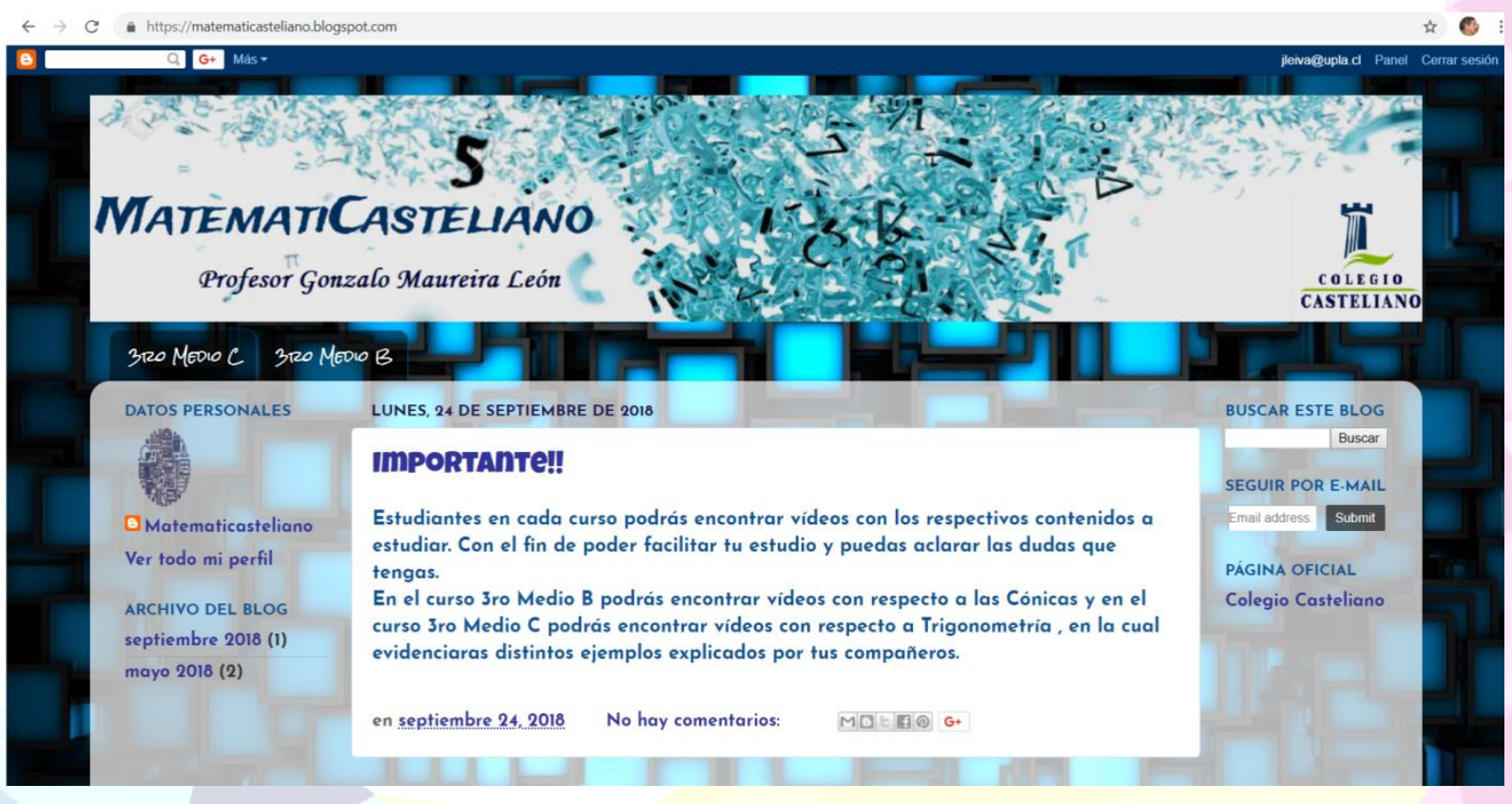

\section{Imagen 1: Blog MatematiCasteliano}

Posteriormente cada uno de los cursos "Tercero medio C" y "Tercero medio B" comenzó a trabajar produciendo materiales (videos) acerca del tema específico (Cónicas o trigonometría) para finalmente entregar el material a profesor quien lo subió al blog en los respectivos espacios creados para cada curso como se observa en la imagen 2 


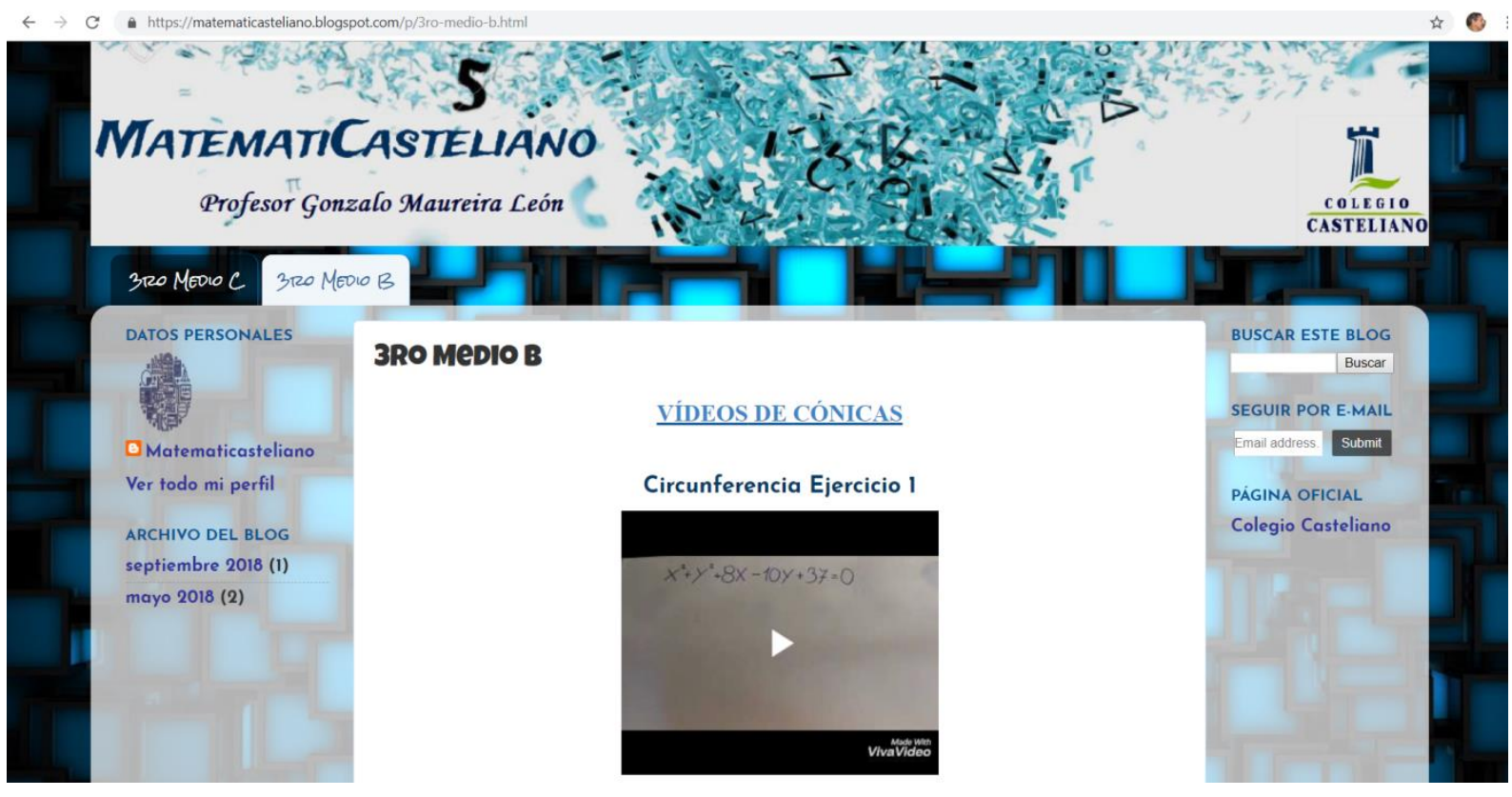

Imagen 2: Vídeos creados por el curso Tercero Medio B

\section{Recopilación de datos: \\ - De los estudiantes}

Para la recopilación de datos se generó un instrumento para estudiantes que fue sometido a juicio de expertos (tres especialistas en TIC), este instrumento finalmente quedo con un total de 10 ítems para los que se generó una escala de Likert de cinco alternativas.

\begin{tabular}{|l|l|l|l|l|l|}
\hline & $\begin{array}{l}\text { Completamente } \\
\text { en desacuerdo }\end{array}$ & $\begin{array}{l}\text { En } \\
\text { desacuerdo }\end{array}$ & $\begin{array}{l}\text { Ni de acuerdo } \\
\text { ni desacuerdo }\end{array}$ & De acuerdo & $\begin{array}{l}\text { Cmpletamente } \\
\text { de acuerdo }\end{array}$ \\
\hline $\begin{array}{l}\text { El trabajo con blog fue una experiencia interesante y } \\
\text { novedosa. }\end{array}$ & & & & & \\
\hline $\begin{array}{l}\text { El uso del blog resultó ser más entretenido para } \\
\text { aprender. }\end{array}$ & & & & & \\
\hline $\begin{array}{l}\text { La metodología usada por el profesor fue más } \\
\text { entretenida y desafiante. }\end{array}$ & & & & & \\
\hline $\begin{array}{l}\text { Me gustaron los materiales creados por mis } \\
\text { compañeros. }\end{array}$ & & & & & \\
\hline $\begin{array}{l}\text { El trabajo con blog me permitió aprender de manera } \\
\text { más entretenida. }\end{array}$ & & & & \\
\hline $\begin{array}{l}\text { La forma en que se utilizó el blog me motivó más } \\
\text { que las formas tradicionales para aprender } \\
\text { matemáticas. }\end{array}$ & & & & & \\
\hline $\begin{array}{l}\text { Me gusto que algo creado por mi apareciera en un } \\
\text { log. }\end{array}$ & & & & & \\
\hline $\begin{array}{l}\text { Me gustaría que los blog fuesen más utilizados por } \\
\text { los profesores. }\end{array}$ & & & & \\
\hline $\begin{array}{l}\text { Los materiales que subieron los compañeros me } \\
\text { ayudaron a aprender }\end{array}$ & & & & \\
\hline $\begin{array}{l}\text { Me gusto que algo creado por mi le sirviera a mis } \\
\text { compañeros para aprender }\end{array}$ & & & & \\
\hline
\end{tabular}




\section{- Del profesor}

Para conocer el significado de la experiencia para el profesor se utilizó la técnica de la entrevista y además se tomó notas de cosas importantes que se conversaron durante todo el proceso. En la entrevista se le realizaron las siguientes consultas:

1.- ¿Pensó en algún momento que utilizaría recursos tecnológicos (TIC) con sus estudiantes?

2.- ¿La experiencia cambió en algo lo que usted pensaba del uso de la tecnología (TIC) en la enseñanza?

3.- ¿Qué dificultades cree usted que dificultan el uso de las TIC por parte de los profesores?

4.- ¿Le gustaría continuar probando con sus estudiantes estrategias metodológicas que utilicen TIC?

\section{RESULTADOS Y DISCUSIÓN}

A partir de las entrevistas realizadas al profesor del curso podemos concluir que todo lo realizado tuvo un significado importante para él desde el punto de vista personal y desde la perspectiva docente: desde el punto de vista personal la experiencia ha significado para el docente mejorar su autoestima en relación al manejo y uso de las tecnologías, ahora se siente más capacitado para utilizarlas y estaría dispuesto a experimentar con otras, la visión que tenía de las tecnologías cambió y ahora piensa que efectivamente pueden mejorar el ambiente de aprendizaje y sirven para innovar en el proceso de enseñanza, siente que su trabajo ha mejorado y que algo que él consideraba lejano como son las tecnologías se pueden aprender con un poco de esfuerzo, sin embargo, el profesor señala que para incorporarlas con eficiencia se requiere de disponer de tiempo y de acompañamiento.

Los estudiantes participaron de la experiencia de manera entusiasta y en general mostraron una buena disposición. Todos los estudiantes estuvieron de acuerdo con que el uso de la tecnología específicamente el blog y los videos les permitió enfrentar el proceso de una forma mucho más entretenida, nunca pensaron que podrían construir videos para colocar en jun blog y para que estudiaran sus compañeros. La mayoría de ellos señalaron que se sintieron muy responsables de construir materiales de buena calidad para que sus compañeros trabajaran y que eso significó que ellos tuviesen que estudiar y entender bien los conceptos relacionados con el trabajo. En general los estudiantes señalaron que sería interesante que actividades como estas se realizaran con otras asignaturas.

\section{CONCLUSIONES}

Las TIC cada día se están posicionando como herramientas para innovar en educación

[180] 
y ya son muy variados los tipos de plataformas utilizadas como medio para poder mejorar las metodologías de aprendizaje, con el fin de desarrollar de una forma concreta, práctica e innovadora cada una de la inteligencia de los estudiantes. Para un docente, lograr implementar nuevas metodologías usando las TIC resulta un gran desafío, y necesariamente requieren de un importante grado de apoyo y acompañamiento para lograr incorporarlas a los ambientes de aprendizaje de manera natural. La tecnología Blog utilizada en este trabajo resultó ser beneficiosa en muchos aspectos, puesto que durante el proceso de creación e implementación fue eficiente y favorable tanto para el profesor, como para los alumnos. Por otra parte el profesor se acomodó bien al uso del blog y seguramente podrá ir disminuyendo el tiempo que necesita para construir material para su clase diaria. Debemos estar cocientes entre docentes que las TIC son una gran herramienta para el aprendizaje y que podemos hacer uso de estas para focalizarnos en tomar decisiones, reflexionar, razonar y resolver problemas. Cualquier asignatura puede beneficiarse de la TIC utilizándolas para generar metodologías más dinámicas y participativas. Es sumamente importante entender que las TIC son sólo un medio al igual que los son el lápiz, papel, instrumentos de dibujo etc. $\mathrm{Y}$ sin un buen diseño pedagógico y la creatividad del profesor no tienen ningún sentido.

Finalmente debemos señalar que la metodología de aprendizaje entre pares puede ser perfectamente mediada con herramientas tecnológicas y resulta ser un proceso interesante para los estudiantes que participan de ella ya que se genera en cada uno de ellos un grado de responsabilidad en relación del aprendizaje que lograrán sus pares.

\section{REFERENCIAS}

ACTIVIDADES PARA EDUCACIÓN INFANTIL. (s. f.). Recuperado 23 de julio, de http://www.actividadeseducainfantil.com/ ,2018.

BARUJEL, A. G. El uso de weblogs en la docencia universitaria. Revista Latinoamericana de Tecnología Educativa - RELATEC, 4(1), 9-24, 2005.

BAUEROVÁ, D., \& SEIN-ECHALUCE, M. L. Herramientas y metodologías para el trabajo cooperativo en red en la Universidad. Revista Interuniversitaria de Formación del Profesorado, 21, 2007.

CHOIS-LENIS P., CASAS-BUSTILLO A., LÓPEZ-HIGUERA A., PRADO-MOSQUERA D., Y CAJAS-PAZ E. Percepciones sobre la tutoría entre pares en escritura académica, Revista Internacional de Investigación en Educación, ISSN-e 2027-1182, Vol. 9, Nº. 19, 2017, págs. 165-184, 2017.

DOMINGO J. El aprendizaje cooperativo, Cuadernos de Trabajo Social, Vol 21, 231-246 ,2008. 
ESOS LOCOS BAJITOS DE INFANTIL. (s. f.). Recuperado 23 de julio, de http://esoslocosbajitosdeinfantil.blogspot.com/,2018.

JOHNSON D., JOHNSON R., HOLUBEC E. El aprendizaje cooperativo en el aula, Editorial Paidos, ISBN 950-12-2144-X, 1999.

LLORENTE, P. A., \& IGLESIAS, E. C. Desarrollo de la competencia digital en la formación inicial del profesorado de Educación Infantil. Píxel-Bit. Revista de Medios y Educación, 0(52), 97-110, 2018.

LOKE A., CHOW F. Asociación de aprendizaje: la experiencia de la tutoría entre pares en estudiantes de enfermería: un estudio cualitativo. International Journal of Nursing Studies 44 (2): 237-44, 2007.

MENESES, E. L. Innovar con blogs en las aulas universitarias. DIM: Didáctica, Innovación y Multimedia, 0(14). Recuperado de https://www.raco.cat/index.php/DIM/article/view/138946, 2009.

MENESES, E. L., \& REGAÑA, C. B. Caminando hacia el software social: una experiencia Universitaria con blogs. Píxel-Bit. Revista de Medios y Educación, 0(32), 67-82, 2008.

OBSERVATORIO DE INNOVACIÓN EDUCATIVA. Radar de innovación educativa 2017, Tecnológico de Monterrey. https://observatorio.itesm.mx/radar-de-innovacin-educativa-2017/ ,2017.

ORIHUELA, J. L. La revolución de los blogs: cuándo las bitácoras se convirtieron en el medio de comunicación de la gente. Madrid: Esfera de los Libros,2006.

PANITZ T. Collaborative versus Cooperative Learning: A Comparison of the Two Concepts Which Will Help Us Understand the Underlying Nature of Interactive Learning, ERIC Number: ED448443, 1-13, 1999.

PEÑA, I., CÓRCOLES, C. P., \& CASADO, C. El Profesor 2.0: docencia e investigación desde la Red. UOC Papers. Revista sobre la sociedad del conocimiento, (3). Recuperado de http://www.redalyc.org/resumen.oa?id=79000307 , 2006.

RECURSOS PARA EDUCACIÓN INFANTIL. (s. f.). Recuperado 23 de julio, de https://marife.wordpress.com/ , 2018.

RODRÍGUEZ M., VÁZQUEZ E. Fortalecer estilos de aprendizaje para aprender a aprender, Revista de Estilos de Aprendizaje Vol 6, No 11, 2013. 